\title{
Neurofibroma subungueal solitário: relato de um caso inédito no sexo masculino
}

\author{
Solitary subungual neurofibroma: a previously unreported finding in a male patient
}

\author{
Dionne de Almeida Stolarczuk ${ }^{1}$ \\ Fernanda da Marca Filgueiras ${ }^{1}$ \\ Solange Cardoso Maciel Costa Silva ${ }^{3}$
}

\author{
Ana Luiza Furtado da Silva ${ }^{1}$ \\ Maria de Fátima Guimarães Scotelaro Alves ${ }^{2}$
}

\begin{abstract}
Resumo: Neurofibroma é uma proliferação hamartomatosa de origem neuromesenquimal. Pode ser encontrado associado à neurofibromatose ou como tumor solitário, sendo sua apresentação subungueal solitária bastante rara. É mais frequente em pacientes do sexo feminino e a cirurgia é o tratamento de escolha. Relatase um caso de um paciente masculino, com lesão tumoral subungueal no pododáctilo, cuja biópsia e imuno-histoquímica foram compatíveis com neurofibroma. Até a presente data, menos de dez casos sobre neurofibromas subungueais sem associação com doença de Von Recklinhausen foram documentados, sendo este o primeiro caso relatado no Brasil e o único no sexo masculino no mundo.

Palavras-chave: Doenças raras; Neoplasias de tecido nervoso; Neurofibroma; Tumores fibrosos solitários

Abstract: A neurofibroma is a hamartomatous proliferation of neuromesenchymal origin. It may be found in combination with neurofibromatosis or in the form of a solitary tumor. Clinical presentation as a solitary subungual tumor is very rare. Neurofibroma is more common in females and surgery is the treatment of choice. The present paper reports the case of a male patient with a subungual tumor on his toe. Biopsy and immunohistochemistry findings were compatible with a neurofibroma. To date, fewer than ten cases of subungual neurofibromas unassociated with von Recklinghausen's disease have been documented, this being the first case to be reported in Brazil and the only report worldwide to have described this condition in a male patient.

Keywords: Neoplasms, nerve tissue; Neurofibroma; Rare diseases; Solitary fibrous tumors
\end{abstract}

\section{INTRODUÇÃO}

Tumores subungueais são responsáveis por diferentes alterações no leito ungueal e, em geral, são subdiagnosticados, tendo em vista suas características inespecíficas. Os neurofibromas, por sua vez, também já foram descritos nesta localização. Podem ocorrer associados à neurofibromatose ou como tumores solitários, sendo incerta sua patogênese nesta situação. Em ambos os casos, esta é uma localização excepcional. Tem crescimento usualmente lento com pouca sintomatologia local e ocorrem tipicamente em mulheres de meia-idade.
Outros tumores como fibroqueratoma, tumor de Köenen, tumor glômico, cisto epidermoide e carcinoma de células escamosas podem simular clinicamente o neurofibroma e devem entrar no diagnóstico diferencial. A cirurgia ungueal auxilia no diagnóstico e na terapêutica, porém, exames de imagens, tais como radiografia e ultrassonografia, também podem auxiliar na visualização do tamanho da lesão e compressão de estruturas ósseas.

Poucos casos de neurofibroma subungueal solitário sem associação com Doença de Von

Recebido em 13.12.2009.

Aprovado pelo Conselho Consultivo e aceito para publicação em 20.06.10.

* Trabalho realizado no Hospital Universitário Pedro Ernesto - Universidade do Estado do Rio de Janeiro (HUPE - UERJ) - Rio de Janeiro (RJ), Brasil.

Suporte financeiro: Nenhum / Conflict of interest: None

Conlito de interesse: Nenhum / Financial funding: None

Pós-graduação em Dermatologia no Hospital Universitário Pedro Ernesto - Universidade do Estado do Rio de Janeiro (HUPE - UERJ) - Especializanda do Hospital Universitário Pedro Ernesto - Universidade do Estado do Rio de Janeiro (HUPE - UERJ) - Rio de Janeiro (RJ), Brasil

Doutorado - Professora-adjunta de Dermatologia do Hospital Universitário Pedro Ernesto - Universidade do Estado do Rio de Janeiro (HUPE - UERJ) - Rio de Janeiro (RJ), Brasil.

Doutorado - Coordenadora da disciplina de Dermatologia do Hospital Universitário Pedro Ernesto - Universidade do Estado do Rio de Janeiro (HUPE - UERJ) - Rio de Janeiro (RJ), Brasil

(C)2011 by Anais Brasileiros de Dermatologia 
Recklinhausen foram descritos na literatura, tornando esta apresentação bastante incomum.

\section{RELATO DO CASO}

Paciente de 51 anos, masculino, branco, natural do Ceará e sem comorbidades. Queixava-se do surgimento de lesão arredondada, sob a lâmina ungueal do quarto pododáctilo direito há aproximadamente 8 meses. Não apresentava alterações prévias na unha e negava trauma ou dor no local. O exame do dígito demonstrava lesão tumoral, de superfície lisa, arredondada, regular, normocrômica, medindo $1,6 \times 1,8 \mathrm{~cm}$ na região subungueal do quarto pododáctilo direito (Figura 1). Restante do exame físico, micológico e laboratorial sem alterações. Radiografia não evidenciou alterações ósseas e na ultrassonografia havia imagem nodular hipoecoica de $15 \times 14 \mathrm{~mm}$, com ausência de fluxo ao Doppler. Foi realizada biópsia, sob anestesia local, com excisão parcial da lesão, que se apresentava polipoide, não encapsulada e brancacenta em seu interior, ocupando a polpa digital (Figura 2). Exame histopatológico evidenciou células fusiformes delgadas com núcleo ondulado em um estroma pálido de colágeno (Figura 3). A imuno-histoquímica foi positiva para $S 100$ e CD34 e negativa para HHF35, desmina e actina 1 a 4. Confirmado o diagnóstico de neurofibroma solitário, o paciente foi submetido a novo procedimento cirúrgico, com incisão da polpa digital e retirada do tumor nesta região e abaixo do leito ungueal. Quando da retirada da lâmina para exploração, observou-se que o tumor estendia-se até a dobra ungueal lateral e foi então realizada sua excisão completa. Até o momento, seis

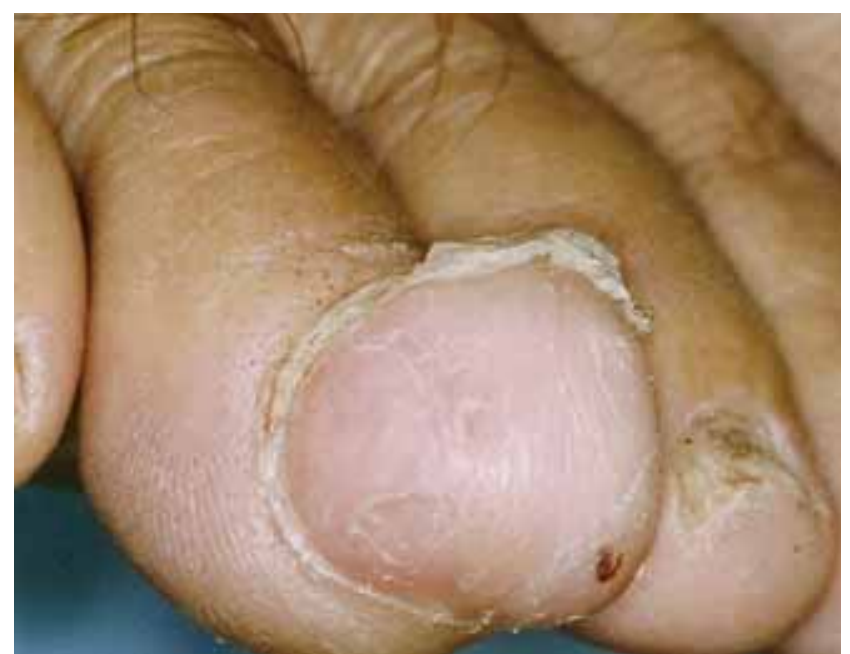

Figura 1: Lesão tumoral, formato arredondado, regular, normocrômica na região subungueal do quarto pododáctilo direito

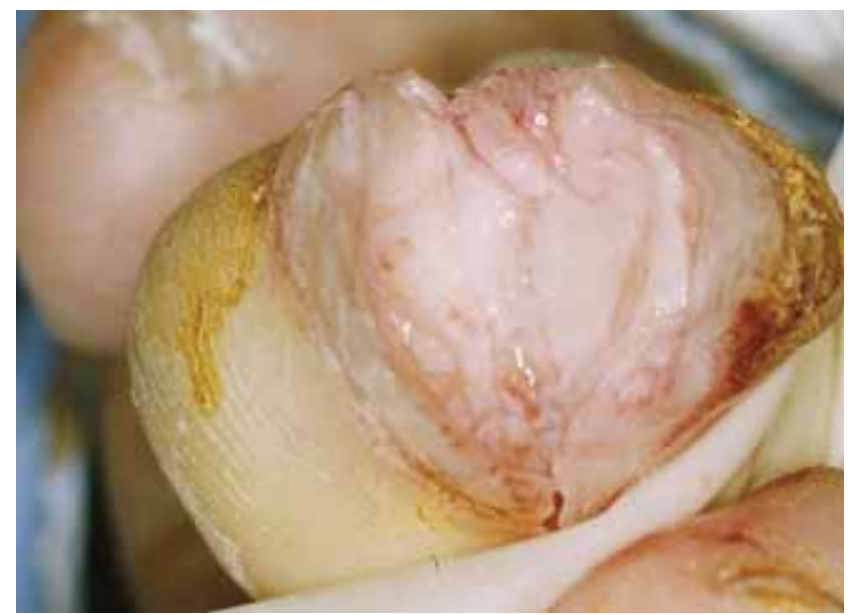

FigURA 2: Lesão polipoide, não encapsulada e brancacenta ocupando a polpa digital

meses após o procedimento cirúrgico, não houve recidiva da doença.

\section{DISCUSSÃO}

Descritos inicialmente por Von Recklinhausen, ${ }^{1}$ os neurofibromas podem ser encontrados de forma múltipla ou solitária, associados ou não à neurofibromatose. São proliferações hamartomatosas de componentes neuromesenquimais, incluindo células de Schwann, fibroblastos, células perineurais e mastócitos. ${ }^{2}$ A proporção das células varia em cada caso. A imuno-histoquímica dos marcadores do tumor pode demonstrar positividade para proteína S100, Melan-A, tirosinase e CD34, entre outros. ${ }^{3}$

Apresentam-se como nódulos circunscritos, normocrômicos e não encapsulados, variando de 0,2

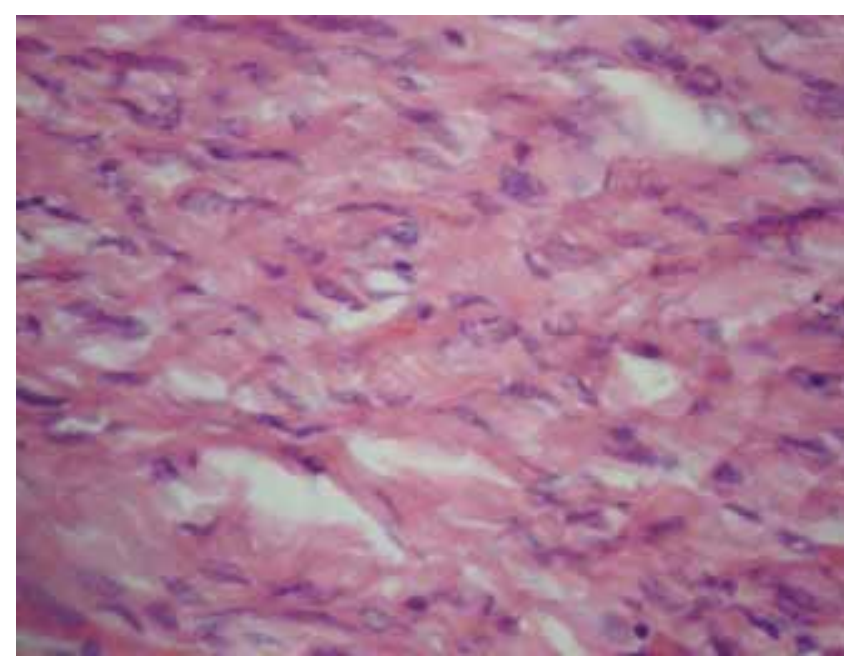

Figura 3: Células fusiformes e delgadas com núcleo ondulado em um estroma pálido de colágeno 
a $2 \mathrm{~cm}$. Os tumores solitários correspondem a 90\% dos casos de neurofibroma, ${ }^{2}$ porém sua apresentação subungueal é bastante rara com poucos casos descritos. Usualmente indolores, podem ocasionar dor, caso ocorra compressão de estruturas próximas. ${ }^{4}$ Quando localizados na região proximal, podem produzir uma depressão longitudinal, mimetizando o tumor de Koenen, ou simular paroníquia crônica, ${ }^{5}$ enquanto na região subungueal podem produzir onicodistrofia ou aumento do volume local. ${ }^{6,4}$ Podem afetar tanto quirodáctilos quanto pododáctilos.

Shelley, em 1986, publicou um dos primeiros relatos de neurofibroma subungueal, em paciente feminina de meia-idade, acometendo segundo quirodáctilo. No entanto, não informava se havia neurofibromatose associada.

Posteriormente, surgiram novos artigos descrevendo casos semelhantes, porém sem evidências de neurofibromatose. Os sintomas descritos variavam desde onicodistrofia,${ }^{4}$ hiperceratose subungueal, ${ }^{6,8}$ aumento do tamanho do dígito afetado até mesmo aumento da curvatura da lâmina ungueal. ${ }^{8}$ Nosso paciente apresentava lesão exclusivamente na região subungueal, sem alterações específicas na lâmina ungueal.

A histopatologia é de fundamental importância para o diagnóstico do neurofibroma, demonstrando uma fina malha fibrilar de células fusiformes em um estroma vascular, fibrótico ou edematoso. ${ }^{2} \mathrm{O}$ principal diagnóstico diferencial histopatológico deve ser feito com o schwannoma, uma vez que as duas patologias apresentam tipos celulares semelhantes e núcleos dispostos em paliçadas podem ocorrer em ambas. No entanto, no neurofibroma, a formação de corpos de Verocay é bastante rara e a proporção de axônios em relação às células de Schwann é menor que $1: 1{ }^{2}$ Além disso, a imuno-histoquímica também pode ajudar na diferenciação dos dois tumores, visto que a reatividade para proteína $S 100$ (positiva nas células de Schwann) é maior nos schwannomas, enquanto nos neurofibromas ela é menos uniforme e menor. Isto ocorre, pois, ao contrário dos schwannomas, que são formados somente por células de Schwann, os neurofibromas consistem de células de Schwann desorganizadas e dispersas associadas a outras células.' O fibromixoma acral superficial, uma neoplasia benigna que eventualmente ocorre na região subungueal, também deve entrar no diagnóstico diferencial. ${ }^{10}$ Apresenta-se como lesão não encapsulada composta por células fusiformes em um estroma mixoide. ${ }^{11}$ Entretanto, a imuno-histoquímica é negativa para proteína $\mathrm{S} 100$, com positividade apenas para $\mathrm{CD} 34 .^{10,11}$
A microscopia eletrônica, descrita em um caso publicado por Niizuma em 1991, evidenciou células de Schwann, fibroblastos, células parenquimatosas e fibrilas de colágeno. ${ }^{12}$ Além disso, a maioria das células parenquimatosas demonstrou positividade para proteína S100, assim como em nosso caso clínico, confirmando a presença de células de Schwann. Outro marcador presente em nossa imunohistoquímica, o $\mathrm{CD} 34$, comprova a natureza neural e fibrosa da lesão, além da presença de vascularização, pois é um marcador de fibroblastos endoneurais ${ }^{10} \mathrm{e}$ células endoteliais ?

Novo relato de caso foi documentado em 2004, em paciente feminina de 50 anos, com lesão no terceiro pododáctilo, sem qualquer sintomatologia clínica. O autor sugere a possibilidade de casos similares serem então subdiagnosticados. ${ }^{13}$

Segundo artigo de revisão publicado por Roldan-Marin em 2006, estes tumores aparecem mais frequentemente em pacientes do sexo feminino de meia-idade, sem evidências de recorrência após a excisão da lesão. ${ }^{14} \mathrm{Em}$ nenhum dos artigos mencionados houve trauma antecedendo o quadro e todos foram relatados em pacientes do sexo feminino. Este fato pode ser decorrente da maior preocupação feminina com a estética corporal, uma vez que o tumor apresenta-se de forma assintomática, apenas com alterações ungueais na grande maioria dos casos. Além disso, em virtude das diferentes formas de apresentação clínica, muitos casos podem ser erroneamente diagnosticados como onicomicose ou trauma, proporcionando um subdiagnóstico das lesões tumorais. Não parece haver influência hormonal no aparecimento ou crescimento da lesão, seja na forma localizada ou até mesmo associada à neurofibromatose.

A radiografia do local acometido é importante para avaliação do acometimento ósseo. No entanto, apenas dois casos evidenciaram compressão falangeana sem invasão óssea. ${ }^{12,4} \mathrm{Da}$ mesma forma, a radiografia de nosso paciente não evidenciou alterações ósseas. A remoção da lâmina ungueal afetada é essencial para visualização e avaliação do tumor, com ressecção curativa da lesão.

Até a presente data, menos de dez casos sobre neurofibromas subungueais sem associação com doença de Von Recklinhausen foram documentados e, após revisão detalhada da literatura disponível, este é o primeiro caso relatado de neurofibroma subungueal solitário no Brasil e o único documentado no sexo masculino no mundo. 


\section{REFERÊNCIAS}

1. Gadelha AR, Akel PBM, Sena RS, Souza MLG, Silva MHCR, Bacry JNA. Neurofibromatose segmentar: relato de caso. An Bras Dermatol. 1999;74:169-71.

2. Argenyi ZB. Neurofibroma. In: Bolognia JL, Lorizzo J, Rapini RP, editors. Dermatology. 2nd ed. London: Mosby. 2008.

3. Fetsch JF, Michal M, Miettinem M. Pigmented (Melanotic) neurofibroma. A clinicopathologic and immunohistochemical analysis of 19 lesions from 17 patients. Am J Surg Pathol. 2000;24:331-43.

4. Dangoisse C, Andre J, De Dobbeleer G, Van Geertruyden J. Solitary subungual neurofibroma. Br J Dermatol. 2000;143:1116-7.

5. Baran R, Dawber RPR, editors. Diseases of the Nails and Their Managemente. $2^{\text {nd }}$ ed. London: Blackwell Science; 1994. p.460.

6. Bhushan M, Telfer NR, Chalmers RJ. Subungual neurofibroma: an unusual cause of nail dystrophy. Br J Dermatol. 1999;140:777-8.

7. Shelley ED, Shelley WB. Exploratory nail plate removal as a diagnosis aid in painful subungual tumors: glomus tumor, neurofibroma and squamous cell carcinoma. Cutis. 1986;38:310-2.

8. Baran R, Haneke E. Subungual myxoid neurofibroma on the thumb. Arch Derm Venereol. 2001;81:210-1.

9. Fcm.unicamp. [internet]. Textos ilustrados de neuropatologia e neuroimagem; c2007. [Acesso: 12 Fev. 2010]. Disponível em:

http://www.fcm.unicamp.br/deptos/anatomia/textosneuro.html

10. Tardío JC. CD34-reactive tumors of the skin. An updated review of an evergrowing list of lesions. J Cutan Pathol. 2009:36:89-102.
11. André J, Theunis A, Richert B, de Saint-Aubain N. Superficial acral fibromyxoma: Clinical and pathological features. Am J Dermatopathol. 2004;26:472-4.

12. Niizuma K, lijima KN. Solitary neurofibroma: a case of subungual neurofibroma on the right third finger. Arch Dermatol Res. 1991:283:13-5.

13. Sugiura K, Sugiura M, Hayakawa R, Kato Y, Sakamoto N, Osada A, et al. Solitary neurofibroma: an uncommon location. Int J Dermatol. 2004;43:451-3.

14. Roldan-Marin R, Dominguez-Cherit J, Vega-Memije ME, Toussaint-Caire S, HojyoTomoka MT, Dominguez-Soto L. Solitary subungual neurofibroma: an uncommon finding and a review of the literature. J drugs Dermatol. 2006;5:672-4.

ENDEREÇO PARA CORRESPONDÊNCIA / MAILING ADDRESS:

Dionne Stolarczuk

Av. General Felicíssimo Cardoso, 835/2007 - Bloco 2

Barra da Tijuca

22631-360 Rio de Janeiro (RJ) - Brasil

E-mail:docsto@botmail.com

Como citar este artigo/How to cite this article: Stolarczuk DA, Silva ALF, Filgueiras FM, Alves MFGS, Silva SCMC. Neurofibroma subungueal solitário: relato de um caso inédito no sexo masculino. An Bras Dermatol. 2011;86(3):569-72. 